\title{
Glider Automorphisms on Some Shifts of Finite Type and a Finitary Ryan's Theorem *
}

\author{
Johan Kopra \\ Department of Mathematics and Statistics, FI-20014 University of Turku, Finland \\ jtjkop@utu.fi
}

\begin{abstract}
For any mixing SFT $X$ containing a fixed point we construct a reversible shift-commuting continuous map (automorphism) which breaks any given finite point of the subshift into a finite collection of gliders traveling into opposing directions. As an application we show that the automorphism group $\operatorname{Aut}(X)$ contains a two-element subset $S$ whose centralizer consists only of shift maps.
\end{abstract}

Keywords: Mixing SFTs, Automorphisms, Cellular automata

\section{Introduction}

Let $X \subseteq A^{\mathbb{Z}}$ be a one-dimensional subshift over a symbol set $A$. If $X$ contains some constant sequence $0^{\mathbb{Z}}(0 \in A)$, we may say that an element $x \in X$ is finite if it differs from $0^{\mathbb{Z}}$ only at finitely many coordinates. In this paper we consider the problem of constructing reversible shift-commuting continuous maps (automorphisms) on $X$ which decompose all finite configurations into collections of gliders traveling into opposing directions. As a concrete example, consider the binary full shift $X=\{0,1\}^{\mathbb{Z}}$ and the map $g=g_{3} \circ g_{2} \circ g_{1}: X \rightarrow X$ defined as follows. In any $x \in X, g_{1}$ replaces every occurrence of 0010 by 0110 and vice versa, $g_{2}$ replaces every occurrence of 0100 by 0110 and vice versa, and $g_{3}$ replace every occurrence of 00101 by 00111 and vice versa. In Figure 1 we have plotted the sequences $x, g(x), g^{2}(x), \ldots$ on consecutive rows for some $x \in X$. It can be seen that the sequence $x$ eventually diffuses into two different "fleets", the one consisting of $1 \mathrm{~s}$ going to the left and the one consisting of 11 s going to the right. It can be proved that this diffusion happens eventually no matter which finite initial point $x \in X$ is chosen. ${ }^{1}$ In Section 3 we construct on all mixing SFTs (that contain the point $0^{\mathbb{Z}}$ ) a glider automorphism with the same diffusion property as the binary automorphism $g$ above.

The existence of a glider automorphism $g$ on a subshift $X$ is interesting, because $g$ can be used to convert an arbitrary finite $x \in X$ into another sequence $g^{t}(x)$ (for some $t \in \mathbb{N}_{+}$) with a simpler structure, which nevertheless contains

\footnotetext{
* The work was partially supported by the Academy of Finland grant 296018 and by the Vilho, Yrjö and Kalle Väisälä Foundation

1 This can be proved along similar lines as in the proof of Lemma 10 and Lemma 11.
} 




Fig. 1. The diffusion of $x \in X$ under the map $g: X \rightarrow X$. White and black squares correspond to digits 0 and 1 respectively.

all the information concerning the original point $x$ because $g$ is invertible. Such maps have been successfully applied to other problems e.g. in $[3,4]$.

We also consider a finitary version of Ryan's theorem. Let $X$ be a mixing SFT and denote the set of its automorphisms by $\operatorname{Aut}(X)$, which we may consider as an abstract group. According to Ryan's theorem [2] the center of the group $\operatorname{Aut}(X)$ is generated by the shift map $\sigma$. There may also be subsets $S \subseteq \operatorname{Aut}(X)$ whose centralizers are generated by $\sigma$. Denote the minimal cardinality of such a finite set $S$ by $k(X)$. In [3] it was proved that $k(X) \leq 10$ when $X$ is the full shift over the four-letter alphabet. In the same paper it is noted that $k(X)$ is an isomorphism invariant of $\operatorname{Aut}(X)$ and therefore computing it could theoretically separate $\operatorname{Aut}(X)$ and $\operatorname{Aut}(Y)$ for some mixing SFTs $X$ and $Y$. We use our glider automorphism construction to prove that $k(X)=2$ for all mixing SFTs that contain the point $0^{\mathbb{Z}}$.

\section{Preliminaries}

A finite set $A$ containing at least two elements (letters) is called an alphabet and the set $A^{\mathbb{Z}}$ of bi-infinite sequences (configurations) over $A$ is called a full shift. Formally any $x \in A^{\mathbb{Z}}$ is a function $\mathbb{Z} \rightarrow A$ and the value of $x$ at $i \in \mathbb{Z}$ is denoted by $x[i]$. It contains finite and one-directionally infinite subsequences denoted by $x[i, j]=x[i] x[i+1] \ldots x[j], x[i, \infty]=x[i] x[i+1] \ldots$ and $x[-\infty, i]=\ldots x[i-1] x[i]$. A factor of $x \in A^{\mathbb{Z}}$ is any finite sequence $x[i, j]$ where $i, j \in \mathbb{Z}$, and we interpret the sequence to be empty if $j<i$. Any finite sequence $w=w[1] w[2] \ldots w[n]$ (also the empty sequence, which is denoted by $\lambda$ ) where $w[i] \in A$ is a word over $A$. The set of all words over $A$ is denoted by $A^{*}$, and the set of non-empty words is $A^{+}=A^{*} \backslash\{\lambda\}$. More generally, for any $L \subseteq A^{*}$, let

$$
L^{*}=\left\{w_{1} \ldots w_{n} \mid n \geq 0, w_{i} \in L\right\} \subseteq A^{*},
$$

i.e. $L^{*}$ is the set of all finite concatenations of elements of $L$. The set of words of length $n$ is denoted by $A^{n}$. For a word $w \in A^{*},|w|$ denotes its length, i.e. $|w|=n \Longleftrightarrow w \in A^{n}$. We say that the word $w \in A^{n}$ occurs in $x \in A^{\mathbb{Z}}$ at position $i$ if $w=x[i] \ldots x[i+n-1]$. We define the shift map $\sigma_{A}: A^{\mathbb{Z}} \rightarrow A^{\mathbb{Z}}$ by $\sigma_{A}(x)[i]=x[i+1]$ for $x \in A^{\mathbb{Z}}, i \in \mathbb{Z}$. The subscript $A$ in $\sigma_{A}$ is typically 
omitted. The set $A^{\mathbb{Z}}$ is endowed with the product topology (with respect to the discrete topology on $A$ ), under which $\sigma$ is a homeomorphism on $A^{\mathbb{Z}}$. Any closed set $X \subseteq A^{\mathbb{Z}}$ such that $\sigma(X)=X$ is called a subshift, and the collection of words appearing as factors of elements of $X$ is the language of $X$, denoted by $L(X)$. The restriction of $\sigma$ to $X$ may be denoted by $\sigma_{X}$, but typically the subscript $X$ is omitted.

If $X \subseteq A^{\mathbb{Z}}$ is a subshift and $z \in X$ is such that $\sigma(z)=z$ (i.e. $z$ is a fixed point), then there exists $a \in A$ such that $z[i]=a$ for all $i \in \mathbb{Z}$. For such subshifts we always fix one such point and denote $a=0, z=0^{\mathbb{Z}}$. Then for $x \in X$ we define its $\operatorname{support} \operatorname{supp}(x)=\{i \in \mathbb{Z} \mid x[i] \neq 0\}$ and say that $x$ is finite if $\operatorname{supp}(x)$ is finite. Finite points $x, y \in X$ with disjoint supports can be glued together; if $\operatorname{supp}(x) \cap \operatorname{supp}(y)=\emptyset$ we define $x \otimes y \in A^{\mathbb{Z}}$ by $(x \otimes y)[i]=x[i]$ when $i \in \operatorname{supp}(x)$ and $(x \otimes y)[i]=y[i]$ otherwise.

Definition 1. A graph is a pair $\mathcal{G}=(V, E)$ where $V$ is a finite set of vertices (or nodes or states) and $E$ is a finite set of edges. Each edge $e \in E$ starts at an initial state denoted by $\iota(e) \in V$ and ends at a terminal state denoted by $\tau(e) \in V$. We say that $e \in A$ is an outgoing edge of $\iota(e)$ and an incoming edge of $\tau(e)$.

A sequence of edges $e[1] \ldots e[n]$ in a graph $\mathcal{G}=(V, E)$ is a path (of length $n$ ) if $\tau(e[i])=\iota(e[i+1])$ for $1 \leq i<n$ and it is a cycle if in addition $\tau(e[n])=\iota(e[1])$. We say that the path starts at $e[1]$ and ends at $e[n]$. A graph $\mathcal{G}$ is primitive if there is $n \in \mathbb{N}_{+}$such that for every $v_{1}, v_{2} \in V$ there is a path of length $n$ starting at $v_{1}$ and ending at $v_{2}$. For any graph $\mathcal{G}=(V, E)$ we call the set

$$
\left\{x \in E^{\mathbb{Z}} \mid \tau(x[i])=\iota(x[i+1])\right\}
$$

(i.e. the set of bi-infinite paths on $\mathcal{G}$ ) the edge subshift of $\mathcal{G}$.

Definition 2. A subshift $X \subseteq A^{\mathbb{Z}}$ is a mixing subshift of finite type (mixing $\mathrm{SFT})$ if it is the edge subshift of a primitive graph $\mathcal{G}=(V, E)$ containing at least two edges (in particular $E \subseteq A$ ).

Example 3. Let $A=\{0, a, b\}$. The graph in Figure 2 defines a mixing SFT $X$ also known as the golden mean shift. A typical point of $X$ looks like

$$
\text { ...000abab0ab00ab000 ... }
$$

i.e. the letter $b$ cannot occur immediately after 0 or $b$ and every occurrence of $a$ is followed by $b$.

Definition 4. An automorphism of a subshift $X \subseteq A^{\mathbb{Z}}$ is a continuous bijection $f: X \rightarrow X$ such that $\sigma \circ f=f \circ \sigma$. We say that $f$ is a radius- $r$ automorphism if $f(x)[0]=f(y)[0]$ for all $x, y \in X$ such that $x[-r, r]=y[-r, r]$ (such $r$ always exists by continuity of $f$ ). The set of all automorphisms of $X$ is a group denoted by $\operatorname{Aut}(X)$. (In the case $X=A^{\mathbb{Z}}$ automorphisms are also known as reversible cellular automata.) 


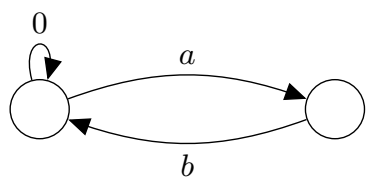

Fig. 2. The golden mean shift.

The centralizer of a set $S \subseteq \operatorname{Aut}(X)$ is

$$
C(S)=\{f \in \operatorname{Aut}(X) \mid f \circ g=g \circ f \text { for every } g \in S\}
$$

and the subgroup generated by $f \in \operatorname{Aut}(X)$ is denoted by $\langle f\rangle$. The following definition is from [3]:

Definition 5. For a subshift $X$, let $k(X) \in \mathbb{N} \cup\{\infty, \perp\}$ be the minimal cardinality of a set $S \subseteq \operatorname{Aut}(X)$ such that $C(S)=\langle\sigma\rangle$ if such a set $S$ exists, and $k(X)=\perp$ otherwise.

The main result of [2] is that $k(X) \neq \perp$ whenever $X$ is a mixing SFT.

We say that subshifts $X \subseteq A^{\mathbb{Z}}$ and $Y \subseteq B^{\mathbb{Z}}$ are conjugate if there is a continuous bijection $\psi: X \rightarrow Y$ such that $\psi \circ \sigma_{X}=\sigma_{Y} \circ \psi$. For conjugate subshifts $X$ and $Y$ it necessarily holds that $k(X)=k(Y)$.

\section{Glider Automorphisms}

In this section we define as a technical tool a subclass of mixing SFTs, and for any subshift $X$ from this class we construct an automorphism which breaks every finite point of $X$ into a collection of gliders traveling in opposite directions.

Note that if $X$ is a mixing SFT with a fixed point $0^{\mathbb{Z}}$, then necessarily in its graph $\mathcal{G}=(V, E)$ it holds that $\tau(0)=\iota(0)$. For such a graph we denote $\mathcal{G}^{\prime}=\left(V, E^{\prime}\right)$ and $E^{\prime}=E \backslash\{0\}$, i.e. we get $\mathcal{G}^{\prime}$ from $\mathcal{G}$ by removing the 0-edge.

Definition 6. A mixing SFT $X$ with a fixed point $0^{\mathbb{Z}}$ and defined by the graph $\mathcal{G}=(V, E)$ is called a 0 -mixing $S F T$ if the graph $\mathcal{G}^{\prime}$ is also primitive and contains at least two edges.

The golden mean shift given by the graph in Figure 2 is an example of a mixing SFT which strictly speaking isn't 0-mixing. Nevertheless, in the following lemma we show that the definition of a 0-mixing SFT is only technical and that it is not an actual restriction.

Lemma 7. Any mixing SFT with a fixed point is conjugate to a 0-mixing SFT.

Proof. Let $X$ be a mixing SFT with a fixed point $0^{\mathbb{Z}}$ defined by the graph $\mathcal{G}=(V, E)$ and let $s=\iota(0)=\tau(0)$. Let $0, a_{1}, \ldots, a_{t}$ be all the outgoing edges of $s$, let $0, b_{1}, \ldots, b_{u}$ be all the incoming edges of $s$ and construct a new graph

$$
\mathcal{H}=\left(V \cup\left\{s^{\prime}\right\}, E \cup\left\{0^{\prime}, b_{1}^{\prime}, \ldots, b_{u}^{\prime}\right\}\right)
$$


with the starting and ending nodes of $e \in E$ the same as in $\mathcal{G}$ with the exception that $\iota\left(a_{i}\right)=s^{\prime}$ for $1 \leq i \leq t$, and additionally $\iota\left(0^{\prime}\right)=s, \tau\left(0^{\prime}\right)=s^{\prime}, \iota\left(b_{j}^{\prime}\right)=\iota\left(b_{j}\right)$ and $\tau\left(b_{j}^{\prime}\right)=s^{\prime}$ for $1 \leq j \leq u .^{2}$ Let $Y$ be the edge subshift of $\mathcal{H}$; it is conjugate with $X$ via the continuous shift-commuting map $\psi: X \rightarrow Y$ defined for $x \in X$, $i \in \mathbb{Z}$ as

$$
\psi(x)[i]=\left\{\begin{array}{l}
0^{\prime} \text { when } x[i]=0 \text { and } x[i+1] \in\left\{a_{1}, \ldots, a_{t}\right\} \\
b_{j}^{\prime} \text { when } x[i]=b_{j} \text { for some } 1 \leq j \leq u \text { and } x[i+1] \in\left\{a_{1}, \ldots, a_{t}\right\}, \\
x[i] \text { otherwise. }
\end{array}\right.
$$
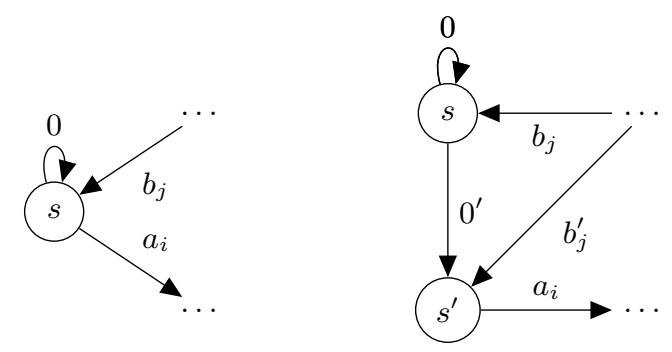

Fig. 3. Splitting the state $s$.

Construct the graphs $\mathcal{G}^{\prime}, \mathcal{H}^{\prime}$ and let $c[1] \ldots c[n] b_{j}$ be a cycle in $\mathcal{G}^{\prime}$ visiting $s$ only at the beginning and ending. Then $c[1] \ldots c[n] b_{j} 0^{\prime}$ and $c[1] \ldots c[n] b_{j}^{\prime}$ are distinct cycles in $\mathcal{H}^{\prime}$ of coprime length, so $\mathcal{H}^{\prime}$ has to be primitive and $Y$ is a 0-mixing SFT.

In the rest of the section we assume that $X$ is a 0 -mixing SFT defined by the graph $\mathcal{G}=(V, E)$. This means that the edge subshift of $\mathcal{G}^{\prime}$ is also a mixing SFT. Denote $s=\iota(0)=\tau(0)$. Let $v_{1}$ be a cycle in $\mathcal{G}^{\prime}$ visiting $s$ only at the beginning and ending, denote $p=\left|v_{1}\right|$ and let $v_{0}=0^{p}$. The words

$$
g_{\ell}=v_{0} v_{1}, \quad g_{r}=v_{1} v_{1}
$$

will be left- and rightbound gliders of the automorphism $g$ defined later. The languages of left- and rightbound gliders are

$$
L_{\ell}=\left(g_{\ell} 00^{*}\right)^{*}, \quad L_{r}=\left(0^{*} 0 g_{r}\right)^{*} .
$$

We denote by ${ }^{\infty} 0$ and $0^{\infty}$ left- and right-infinite sequences of zeroes and define the glider fleet sets

$$
\mathrm{GF}_{\ell}={ }^{\infty} 0\left(g_{\ell} 00^{*}\right)^{*} 0^{\infty} \quad \mathrm{GF}_{r}={ }^{\infty} 0\left(0^{*} 0 g_{r}\right)^{*} 0^{\infty} \quad \mathrm{GF}=\mathrm{GF}_{\ell} \cup \mathrm{GF}_{r}
$$

\footnotetext{
${ }^{2}$ In other words we have performed an elementary state splitting of $\mathcal{G}$ at state $s$. State splitting is a well-known method to produce conjugate subshifts, see e.g. Chapter 2.4 of $[1]$.
} 
(note that these consist of finite configurations).

Denote $u^{\prime}=v_{1} v_{1} v_{1}$ and let $n \in \mathbb{N}_{+}$be a mixing constant of $\mathcal{G}^{\prime}$ (i.e. a number such that for every $n^{\prime} \geq n$ and $s_{1}, s_{2} \in V$ there is a path of length $n^{\prime}$ in $\mathcal{G}^{\prime}$ from $s_{1}$ to $s_{2}$ ) chosen such that $n \geq\left|u^{\prime}\right|=3 p$. For every $a \in E$ we may choose some path $w_{a} \in E^{\prime 2 n}$ in $\mathcal{G}^{\prime}$ such that $w_{a}$ begins with $u^{\prime}$ and $0 w_{a} a \in L(X)$. For every $a \in E$ let $W_{a}^{\prime}=\left\{w_{a, 1}, \ldots, w_{a, k_{a}}\right\} \subseteq E^{\prime 2 n}$ be the paths of length $2 n$ in $\mathcal{G}^{\prime}$ such that $w_{a, i}$ does not have a prefix $u^{\prime}$ and $0 w_{a, i} a \in L(X)$ for $1 \leq i \leq k_{a}$, and let $W_{a}=W_{a}^{\prime} \cup\left\{w_{a}\right\}$. Let $U^{\prime}=\left\{u_{1}^{\prime}, \ldots, u_{k}^{\prime}\right\} \subseteq\left(E^{\prime}\right)^{+}$be the cycles from $s$ to $s$ (which may visit $s$ several times) of length at most $2 n-1 \geq 5 p$ which are different from $v_{1}$ and $v_{1} v_{1}$ and do not have $u^{\prime}$ as a prefix. Finally, these words are padded to constant length; $u=0^{2 n-1-\left|u^{\prime}\right|} u^{\prime}$ and $u_{i}=0^{2 n-1-\left|u_{i}^{\prime}\right|} u_{i}^{\prime}$. The words in $W_{a}$ and $U^{\prime}$ are chosen so as to allow the following structural definition.

Definition 8. Assume that $x \notin \mathrm{GF}_{\ell}$ is a non-zero finite element of $X$. Then there is a maximal $i \in \mathbb{Z}$ such that

$$
x[-\infty, i-1] \in{ }^{\infty} 0 L_{\ell},
$$

and there is a unique word $w \in\left\{v_{1} 0\right\} \cup\left\{v_{1} v_{1} 0\right\} \cup\left\{u^{\prime}\right\} \cup\left(U^{\prime} 0\right) \cup\left(\bigcup_{a \in E} W_{a}^{\prime} a\right)$ such that $w$ is a prefix of $x[i, \infty]$. If $w=v_{1} v_{1} 0$ or $w \in U^{\prime} 0$, let $j=i+|w|-1$ and otherwise let $j=i+\left|v_{1}\right|$. We say that $x$ is of left bound type $(w, j)$ and that it has left bound $j$ (note that $j>i$ ).

Similarly, if $x \notin \mathrm{GF}_{r}$ is a non-zero finite element of $X$, then there is a minimal $j \in \mathbb{Z}$ such that

$$
x[j+1, \infty] \in L_{r} 0^{\infty}
$$

and we say that $x$ has right bound $j$.

The point of this definition is that if $x$ is of left bound type $(w, j)$, then the glider automorphism $g$ defined later will create a new leftbound glider at position $j$ and break it off from the rest of the configuration.

We define four maps $g_{1}, g_{2}, g_{3}, g_{4}: X \rightarrow X$ as follows. In any $x \in X$,

- $g_{1}$ replaces every occurrence of $0\left(v_{0} v_{1}\right) 0$ by $0\left(v_{1} v_{1}\right) 0$ and vice versa

- $g_{2}$ replaces every occurrence of $0\left(v_{1} v_{0}\right) 0$ by $0\left(v_{1} v_{1}\right) 0$ and vice versa

- $g_{3}$ replaces every occurrence of $0 v_{0}\left(v_{1} v_{0} v_{1}\right)$ by $0 v_{0}\left(v_{1} v_{1} v_{1}\right)$ and vice versa

- $g_{4}$ replaces every occurrence of $0 w_{a} a, 0 w_{a, i} a$ and $0 w_{a, k_{a}} a$ by $0 w_{a, 1} a, 0 w_{a, i+1} a$ and $0 w_{a} a$ respectively (for $a \in E$ and $1 \leq i<k_{a}$ ) and every occurrence of $0 u 0,0 u_{i} 0$ and $0 u_{k} 0$ by $0 u_{1} 0,0 u_{i+1} 0$ and $0 u 0$ respectively (for $1 \leq i<k$ ).

It is easy to see that these maps are well defined automorphisms of $X$. The glider automorphism $g: X \rightarrow X$ is defined as the composition $g_{4} \circ g_{3} \circ g_{2} \circ g_{1}$. The name is partially justified by the following lemma.

Lemma 9. If $x \in \mathrm{GF}_{\ell}$ (resp. $x \in \mathrm{GF}_{r}$ ), then $g(x)=\sigma^{p}(x)$ (resp. $g(x)=$ $\left.\sigma^{-p}(x)\right)$.

Proof. Assume that $x \in \mathrm{GF}_{\ell}$ (the proof for $x \in \mathrm{GF}_{r}$ is similar) and assume that $i \in \mathbb{Z}$ is some position in $x$ where $g_{\ell}$ occurs. Then

$$
x[i-1, i+2 p]=0 g_{\ell} 0=0\left(v_{0} v_{1}\right) 0
$$




$$
\begin{aligned}
& g_{1}(x)[i-1, i+2 p]=0\left(v_{1} v_{1}\right) 0 \\
& g_{2}\left(g_{1}(x)\right)[i-p-1, i+p]=0 v_{0}\left(v_{1} 0\right)=0 g_{\ell} 0 \\
& \left.g(x)=g_{4}\left(g_{3}\left(g_{2}\left(g_{1}(x)\right)\right)\right)=g_{2}\left(g_{1}(x)\right)\right),
\end{aligned}
$$

so every glider has shifted by distance $p$ to the left and $g(x)=\sigma^{p}(x)$.

In fact, the previous lemma would hold even if $g$ were replaced by $g_{2} \circ g_{1}$. The role of the part $g_{4} \circ g_{3}$ is, for a given finite point $x \in X$, to "erode" non-zero non-glider parts of $x$ from the left and to turn the eroded parts into new gliders. This is the content of the following lemmas.

Lemma 10. Assume that $x \in X$ has left bound $j$. Then there exists $t \in \mathbb{N}_{+}$ such that the left bound of $g^{t}(x)$ is strictly greater than $j$.

Proof. Let $x \in X$ be of left bound type $(w, j)$ with $w \in\left\{v_{1} 0\right\} \cup\left\{v_{1} v_{1} 0\right\} \cup\left\{u^{\prime}\right\} \cup$ $\left(U^{\prime} 0\right) \cup\left(\bigcup_{a \in E} W_{a}^{\prime} a\right)$. The gliders to the left of the occurrence of $w$ near $j$ move to the left at constant speed $p$ under action of $g$ without being affected by the remaining part of the configuration.

Case 1. Assume that $w=v_{1} v_{1} 0$. Then $g_{1}(x)[j-(p+1), j]=0 v_{1} 0$. If $g_{1}(x)[j-$ $(p+1), j+p]=0 v_{1} v_{0} 0$, then $g(x)[j-(p+1), j+p]=g_{2}\left(g_{1}(x)\right)[j-(p+$ $1), j+p]=0 v_{1} v_{1} 0, g(x)$ is of left bound type $\left(v_{1} v_{1} 0, j+p\right)$ and we are done. Otherwise $g_{2}\left(g_{1}(x)\right)[j-(p+1), j]=0 v_{1} 0$. Denote $y=g_{3}\left(g_{2}\left(g_{1}(x)\right)\right)$. If $y[j-(p+1), j]=0 v_{1} 0$, then $g(x)=g_{4}(y)$ is of left bound type $\left(v_{1} 0, j\right)$ and we proceed as in Case 3. Otherwise $y[j-(p+1), j+(2 p-1)]=0 u^{\prime}$. If $y[(j+2 p)-2 n, j+2 p]=0 u 0$, then $g(x)[(j+2 p)-2 n, j+2 p]=0 u_{1} 0, g(x)$ is of left bound type $\left(u_{1}^{\prime} 0, j+2 p\right)$ and we are done. On the other hand, if $y[(j+2 p)-2 n, j+2 p] \neq 0 u 0$, then $g(x)$ is of left bound type $\left(w^{\prime}, j\right)$ for some $w^{\prime} \in W_{a}^{\prime} a \cup\left\{u^{\prime}\right\}(a \in E)$ and we proceed as in Case 4 or Case 5 .

Case 2. Assume that $w=u_{i}^{\prime} 0$ for $1 \leq i \leq k$. There is a minimal $t \in \mathbb{N}$ such that $g_{3}\left(g_{2}\left(g_{1}\left(g^{t}(x)\right)\right)\right)[j-2 n, j]=0 u_{i} 0$. Because $g^{t+k-i+1}(x)[j-2 n, j]=0 u 0$, it follows that $y=g^{t+k-i+1}(x)$ is of left bound type $\left(u^{\prime}, j-2 p\right)$. Then $g(y)[j-5 p, j]=g_{3}\left(g_{2}\left(g_{1}(y)\right)\right)[j-5 p, j]=v_{0}\left(v_{0} v_{1}\right) v_{0} v_{1} 0$ is of left bound type $\left(v_{1} 0, j\right)$ and we proceed as in Case 3.

Case 3. Assume that $w=v_{1} 0$. Then $x[j-(2 p+1), j] \neq 0 v_{0} v_{1} 0=0 g_{\ell} 0$ because otherwise the left bound of $x$ would already be greater than $j$, so $g_{1}(x)[j-$ $(p+1), j]=0 v_{1} 0$. If moreover $g_{1}(x)[j-(p+1), j+p]=0 v_{1} v_{0} 0$, then $g(x)[j-$ $(p+1), j+p]=g_{2}\left(g_{1}(x)\right)[j-(p+1), j+p]=0\left(v_{1} v_{1}\right) 0$ so $g(x)$ is of left bound type $\left(v_{1} v_{1} 0, j+p\right)$ and we are done. Let us therefore assume that $g_{1}(x)[j-(p+1), j+p] \neq 0 v_{1} v_{0} 0$, in which case $g_{2}\left(g_{1}(x)\right)[j-(2 p+1), j]=$ $0 v_{0} v_{1} 0$.

If $g_{2}\left(g_{1}(x)\right)[j-(2 p+1), j+2 p-1] \neq 0 v_{0} v_{1} v_{0} v_{1}$, then $g(x)[j-(2 p+$ $1), j]=g_{3}\left(g_{2}\left(g_{1}(x)\right)\right)[j-(2 p+1), j]=0 v_{0} v_{1} 0$. The left bound of $g(x)$ is now greater than $j$ and we are done. Otherwise $g_{3}\left(g_{2}\left(g_{1}(x)\right)\right)[j-(2 p+1), j+$ $2 p-1]=0 v_{0} u^{\prime}$. If $g_{3}\left(g_{2}\left(g_{1}(x)\right)\right)[(j+2 p)-2 n, j+2 p]=0 u 0$, then $g(x)[(j+$ $2 p)-2 n, j+2 p]=0 u_{1} 0$ and the left bound of $g(x)$ equals $j+2 p$. Finally, if $g_{3}\left(g_{2}\left(g_{1}(x)\right)\right)[(j+2 p)-2 n, j+2 p] \neq 0 u 0$, then $g(x)$ is of left bound type 
$\left(w^{\prime}, j\right)$ for some $w^{\prime} \in W_{a}^{\prime} a \cup\left\{u^{\prime}\right\}(a \in E)$ and we proceed as in Case 4 or Case 5.

Case 4. Assume that $w=w_{a, i} a$ for $a \in E$ and $1 \leq i \leq k_{a}$. Then $g^{k_{a}-i+1}(x)[j-$ $p, j+(2 p-1)]=u^{\prime}$ and we proceed as in Case 5 .

Case 5. Assume that $w=u^{\prime}$. Then $g_{2}\left(g_{1}(x)\right)[j-(2 p+1), j+(2 p-1)]=0 v_{0} u^{\prime}$, $g_{3}\left(g_{2}\left(g_{1}\right)\right)[j-(2 p+1), j+(2 p-1)]=0 v_{0} v_{1} v_{0} v_{1}$ and the left bound of $g(x)$ is at least $j+2 p$.

Lemma 11. Assume that $x \in X$ has right bound $j$. Then there exists $t \in \mathbb{N}_{+}$ such that the right bound of $g^{t}(x)$ is strictly less than $j$.

Proof. Let us assume to the contrary that the right bound of $g^{t}(x)$ is at least $j$ for every $t \in \mathbb{N}_{+}$.

Assume first that the right bound of $g^{t}(x)$ is equal to $j$ for every $t \in \mathbb{N}_{+}$. By the previous lemma there is $t \in \mathbb{N}_{+}$such that the left bound of $g^{t}(x)$ is at least $j+3 n$, which means that $g^{t}(x)$ contains only $g_{\ell^{-}}$gliders to the left of $j+n$ and only $g_{r}$-gliders to the right of $j$. This can happen only if $g^{t}(x)[j+1, n-1]=0^{n-1}$. Then the right bound of $g^{t+1}(x)$ is at least $j-p$, a contradiction.

Assume then that the right bound of $g^{t}(x)$ is strictly greater that $j$ for some $t \in \mathbb{N}_{+}$and fix the minimal such $t$. This can happen only if $g_{1}\left(g^{t-1}(x)\right)[j-p, j+$ $p+1]=0 v_{1} v_{0} 0$, in which case $g^{t}(x)[j-p, j+p+1]=0 v_{1} v_{1} 0=0 g_{r} 0$. But then the right bound of $g^{t}(x)$ is less than $j-p$, a contradiction.

Together these two lemmas yield the following theorem.

Theorem 12. If $x \in X$ is a finite configuration, then for every $N \in \mathbb{N}$ there exists $t \in \mathbb{N}$ such that $g^{t}(x)[-N, N]=0^{2 N+1}, g^{t}(x)[\infty,-(N+1)] \in{ }^{\infty} 0 L_{\ell}$ and $g^{t}(x)[N+1, \infty] \in L_{r} 0^{\infty}$.

\section{Finitary Ryan's Theorem}

In this section we prove a finitary version of Ryan's theorem. The idea is that only very specific automorphisms commute with the glider map $g: X \rightarrow X$ defined in the previous section, so it will be relatively easy to choose another automorphism $f$ on $X$ such that only powers of the shift map commute with both $g$ and $f$. We make a simple choice of such $f$.

First we define maps $f_{1}, f_{2}: X \rightarrow X$ for a 0 -mixing SFT $X$ as follows. In any $x \in X$,

- $f_{1}$ replaces every occurrence of $0\left(v_{1} v_{1}\right) v_{0} v_{0} v_{0}\left(v_{1}\right) 0$ by $0\left(v_{1} v_{1}\right) v_{0} v_{0}\left(v_{1}\right) v_{0} 0$ and vice versa

- $f_{2}$ replaces every occurrence of $0\left(v_{1} v_{1}\right) v_{0} v_{0}\left(v_{1}\right) 0$ by $0 v_{0}\left(v_{1} v_{1}\right) v_{0}\left(v_{1}\right) 0$ and vice versa, 
where $v_{0}$ and $v_{1}$ are as in the previous section. It is easy to see that these maps are well defined automorphisms of $X$. The automorphism $f: X \rightarrow X$ is then defined as the composition $f_{2} \circ f_{1}$. The map $f$ has two important properties. First, it replaces any occurrence of $0\left(v_{1} v_{1}\right) v_{0} v_{0} v_{0}\left(v_{1}\right) 0$ by $0 v_{0}\left(v_{1} v_{1}\right) v_{0}\left(v_{1}\right) v_{0} 0$. Second, if $x \in X$ is a configuration containing only gliders $g_{\ell}$ and $g_{r}$ and every occurrence of $g_{\ell}$ is sufficiently far from every occurrence of $g_{r}$, then $f(x)=x$.

To prove our main result we need the following lemma.

Lemma 13 ([3], Lemma 7.5). If $X$ is a mixing SFT containing a fixed point $0^{\mathbb{Z}}$ and $h: X \rightarrow X$ is an automorphism which is not a power of $\sigma$, then there exists a finite configuration $x \neq 0^{\mathbb{Z}}$ such that $h(x) \notin \mathcal{O}(x) \doteqdot\left\{\sigma^{i}(x) \mid i \in \mathbb{Z}\right\}$.

Theorem 14. Let $X \subseteq A^{\mathbb{Z}}$ be a 0 -mixing SFT and $g, f: X \rightarrow X$ as above. The only automorphisms of $X$ which commute with both $g$ and $f$ are powers of $\sigma$.

Proof. Assume to the contrary that $h: X \rightarrow X$ is a radius- $r$ automorphism whose inverse is also a radius- $r$ automorphism and which commutes with $g$ and $f$ but is not a power of $\sigma$. Let us first show that $h\left(0^{\mathbb{Z}}\right)=0^{\mathbb{Z}}$. Namely, if it were that $h\left(0^{\mathbb{Z}}\right)=a^{\mathbb{Z}}$, for some $a \in A \backslash\{0\}$, consider $x=\ldots 000 g_{\ell} 000 \ldots$ with the glider $g_{\ell}$ at the origin and note that $h(x)[i] \neq a$ for some $-r \leq i \leq(2 p-1)+r$ (recall: $\left.g_{\ell}=v_{0} v_{1},\left|v_{0}\right|=\left|v_{1}\right|=p\right)$ and $h(x)[-\infty, i-j p]=\ldots$ aaa for some $j \in \mathbb{N}_{+}$. Then $g^{t}(h(x))[-\infty, i-j p]=\ldots a a a$ for every $t \in \mathbb{Z}$ but $h\left(g^{j}(x)\right)[i-j p]=$ $h\left(\sigma^{j p}(x)\right)[i-j p]=h(x)[i] \neq a$, contradicting the commutativity of $h$ and $g$. Thus $h$ maps finite configurations to finite configurations.

We have $h\left(\mathrm{GF}_{\ell}\right) \subseteq \mathrm{GF}_{\ell}$. To see this, assume to the contrary that there exists $x \in \mathrm{GF}_{\ell}$ such that $h(x) \notin \mathrm{GF}_{\ell}$. Recall that $g$ is reversible and $g\left(\mathrm{GF}_{\ell}\right)=\mathrm{GF}_{\ell}$, so $g^{t}(h(x)) \notin \mathrm{GF}_{\ell}$ for all $t \in \mathbb{N}$. Combining this with Theorem 12 it follows that $g^{t}(h(x))$ contains an occurrence of $0 v_{1} v_{1} 0$ to the right of coordinate $r$ for all sufficiently large $t$ and therefore $h^{-1}\left(g^{t}(h(x))\right)[i]$ is non-zero for some $i \geq 0$ which depends on $t$. This contradicts the fact that $h^{-1}\left(g^{t}(h(x))\right)[i]=g^{t}(x)[i]=0$ for all $i \geq 0$ given that $t$ is sufficiently big. Similarly $h\left(\mathrm{GF}_{r}\right) \subseteq \mathrm{GF}_{r}$.

For any finite $x \neq 0^{\mathbb{Z}}$ define its left and right offsets

$$
\begin{aligned}
& \operatorname{off}_{\ell}(x)=\min \{\operatorname{supp}(h(x))\}-\min \{\operatorname{supp}(x)\}, \\
& \operatorname{off}_{r}(x)=\max \{\operatorname{supp}(h(x))\}-\max \{\operatorname{supp}(x)\} .
\end{aligned}
$$

For all nonzero $x_{\ell} \in \mathrm{GF}_{\ell}$ and $x_{r} \in \mathrm{GF}_{r}$ we have off $\left(_{\ell}\left(x_{\ell}\right)-\mathrm{off}_{r}\left(x_{r}\right)=0\right.$. If this did not hold, we could assume without loss of generality that off ${ }_{\ell}\left(x_{\ell}\right)-$ off $_{r}\left(x_{r}\right)>0$ (by replacing $h$ with $h^{-1}$ if necessary) and that $\min \left\{\operatorname{supp}\left(x_{\ell}\right)\right\}=(r+2) p$, $\max \left\{\operatorname{supp}\left(x_{r}\right)\right\}=-(r+1) p-1$ (by shifting $x_{\ell}$ and $x_{r}$ suitably). Then consider $x=x_{r} \otimes x_{\ell}$ and note that from $\min \left\{\operatorname{supp}\left(x_{\ell}\right)\right\}=(r+2) p>r, \max \left\{\operatorname{supp}\left(x_{r}\right)\right\}=$ $-(r+1) p-1<-r$ it follows that $h(x)=h\left(x_{r}\right) \otimes h\left(x_{\ell}\right)$. Then $g^{r}(x)[-3 p-1,3 p]=$ $0\left(v_{1} v_{1}\right) v_{0} v_{0} v_{0}\left(v_{1}\right) 0$ and $g^{r}(h(x))$ contains no occurrence of the words mentioned in the definition of $f_{1}$ and $f_{2}$ by the assumption off $\left(_{\ell}\left(x_{\ell}\right)-\operatorname{off}_{r}\left(x_{r}\right)>0\right.$, so $f\left(g^{r}(x)\right) \neq g^{r}(x)$ and $f\left(g^{r}(h(x))\right)=g^{r}(h(x))$. Now

$$
x \neq g^{-r}\left(f\left(g^{r}(x)\right)\right)=h^{-1}\left(g^{-r}\left(f\left(g^{r}(h(x))\right)\right)\right)
$$




$$
=h^{-1}\left(g^{-r}\left(g^{r}(h(x))\right)\right)=x,
$$

a contradiction. It also follows that there is a fixed $s \in \mathbb{Z}$ such that $\operatorname{off}_{\ell}\left(x_{\ell}\right)=$ $\operatorname{off}_{r}\left(x_{r}\right)=s$ for all nonzero $x_{\ell} \in \mathrm{GF}_{\ell}, x_{r} \in \mathrm{GF}_{r}$.

If $x_{\ell} \in \mathrm{GF}_{\ell}$ and $x_{r} \in \mathrm{GF}_{r}$ are configurations containing exactly one occurrence of $g_{\ell}$ and $g_{r}$ respectively, then $h\left(x_{\ell}\right)=\sigma^{-s}\left(x_{\ell}\right)$ and $h\left(x_{r}\right)=\sigma^{-s}\left(x_{\ell}\right)$. To see this, assume to the contrary (without loss of generality) that $\min \left\{\operatorname{supp}\left(x_{\ell}\right)\right\}=$ $(r+2) p$ (i.e. the occurrence of $g_{\ell}$ in $x$ is at $\left.(r+1) p\right), \max \left\{\operatorname{supp}\left(x_{r}\right)\right\}=-(r+1) p-1$ and $h\left(x_{\ell}\right)[(r+1) p+s,(r+3) p-1+s]=h\left(x_{\ell}\right)[(r+1) p+s+k,(r+3) p-1+s+k]=g_{\ell}$ for some $k>2 p$ (i.e. $h\left(x_{\ell}\right)$ contains at least two occurrences of $g_{\ell}$, the case in which $h\left(x_{r}\right)$ contains at least two occurrences of $g_{r}$ being similar). Then consider $x=x_{r} \otimes x_{\ell}$ and note that

$$
\begin{aligned}
& g^{r}(x)[-3 p-1,3 p]=0\left(v_{1} v_{1}\right) v_{0} v_{0} v_{0}\left(v_{1}\right) 0 \\
& f\left(g^{r}(x)\right)[-3 p-1,3 p]=0 v_{0}\left(v_{1} v_{1}\right) v_{0}\left(v_{1}\right) v_{0} 0 \\
& g^{-1}\left(f\left(g^{r}(x)\right)\right)[-3 p-1,3 p]=0\left(v_{1} v_{1}\right) v_{0} v_{0} v_{0}\left(v_{1}\right) 0 \\
& g^{-(r+1)}\left(f\left(g^{r}(x)\right)\right)=x,
\end{aligned}
$$

therefore also $h(x)=g^{-(r+1)}\left(f\left(g^{r}(h(x))\right)\right) \doteqdot y$. On the other hand,

$$
\begin{aligned}
& f\left(g^{r}(h(x))\right)[p+s+k, 3 p-1+s+k] \\
= & g^{r}(h(x))[p+s+k, 3 p-1+s+k]=g_{\ell} \\
& g^{-(r+1)}\left(f\left(g^{r}(h(x))\right)\right)[(r+2) p+s+k,(r+4) p-1+s+k]=g_{\ell},
\end{aligned}
$$

so in particular $y[(r+2) p+s+k,(r+3) p-1+s+k]=v_{0}$. Because we assumed that

$$
\begin{aligned}
& h(x)[(r+2) p+s+k,(r+3) p-1+s+k] \\
= & h\left(x_{\ell}\right)[(r+2) p+s+k,(r+3) p-1+s+k]=v_{1},
\end{aligned}
$$

it follows that $h(x) \neq y$, a contradiction.

By Lemma 13 there exists a finite configuration $x \neq 0^{\mathbb{Z}}$ such that $h(x) \notin \mathcal{O}(x)$ and $h(x)$ is finite. Use Theorem 12 to get $t \in \mathbb{N}$ such that $g^{t}(x)[-r, r]=0^{2 r+1}$ and $g^{t}(x)=y_{\ell} \otimes y_{r}$ where $y_{\ell} \in \mathrm{GF}_{\ell}$ has $\max \left\{\operatorname{supp}\left(y_{\ell}\right)\right\}<-r$ and $y_{r} \in \mathrm{GF}_{r}$ has $\min \left\{\operatorname{supp}\left(y_{r}\right)\right\}>r$ (it is possible that either $y_{\ell}$ or $y_{r}$ is equal to $0^{\mathbb{Z}}$ ). Then also $h\left(g^{t}(x)\right)=h\left(y_{\ell}\right) \otimes h\left(y_{r}\right) \notin \mathcal{O}\left(g^{t}(x)\right)$, and combining this with off $\ell\left(y_{\ell}\right)=\operatorname{off}_{r}\left(y_{r}\right)$ it follows that $h\left(y_{\ell}\right) \notin \mathcal{O}\left(y_{\ell}\right)$ or $h\left(y_{r}\right) \notin \mathcal{O}\left(y_{r}\right)$. Without loss of generality assume that $h\left(y_{\ell}\right) \notin \mathcal{O}\left(y_{\ell}\right)$ (the case $h\left(y_{r}\right) \notin \mathcal{O}\left(y_{r}\right)$ is similar), that $y_{\ell}$ contains a minimal number of occurrences of $g_{\ell}$ (at least two by the previous paragraph) and that the distance from the leftmost $g_{\ell}$ to the second-to-leftmost $g_{\ell}$ in $y_{\ell}$ is maximal (at most $2 r+2 p$ since otherwise by dropping the leftmost $g_{\ell}$ we would get a new configuration $y_{\ell}^{\prime}$ such that $h\left(y_{\ell}^{\prime}\right) \notin \mathcal{O}\left(y_{\ell}^{\prime}\right)$, contradicting the minimal number of occurrences of $g_{\ell}$ in $y_{\ell}$ ). Let $x_{r} \in \mathrm{GF}_{r}$ contain exactly one occurrence of $g_{r}$ and assume that $\min \left\{\operatorname{supp}\left(y_{\ell}\right)\right\}=(r+2) p, \max \left\{\operatorname{supp}\left(x_{r}\right)\right\}=-(r+1) p-1$. 
Decompose $y_{\ell}=x_{\ell} \otimes x_{\ell}^{\prime}$ so that $x_{\ell}$ contains only the leftmost $g_{\ell}$ from $y_{\ell}$ and $x_{\ell}^{\prime}$ contains all the other occurrences of $g_{\ell}$ from $y_{\ell}$. In a similar way as in the previous paragraph we see that

$$
g^{-(r+1)}\left(f\left(g^{r}\right)\right)\left(x_{r} \otimes y_{\ell}\right)=x_{r} \otimes\left(x_{\ell} \otimes\left(\sigma^{-p}\left(x_{\ell}^{\prime}\right)\right)\right) .
$$

Denote $h^{\prime}=\left(g^{-(r+1)} \circ f \circ g^{r}\right)^{-1}$. By the maximality of distance from the leftmost $g_{\ell}$ to the second-to-leftmost $g_{\ell}$ in $y_{\ell}$ we know that

$$
h\left(x_{\ell} \otimes\left(\sigma^{-p}\left(x_{\ell}^{\prime}\right)\right)\right) \in \mathcal{O}\left(x_{\ell} \otimes\left(\sigma^{-p}\left(x_{\ell}^{\prime}\right)\right)\right),
$$

and this is possible only if

$$
\begin{aligned}
& h\left(x_{\ell} \otimes\left(\sigma^{-p}\left(x_{\ell}^{\prime}\right)\right)\right)=\sigma^{-s}\left(x_{\ell} \otimes\left(\sigma^{-p}\left(x_{\ell}^{\prime}\right)\right)\right), \\
& h\left(x_{r} \otimes x_{\ell} \otimes\left(\sigma^{-p}\left(x_{\ell}^{\prime}\right)\right)\right)=\sigma^{-s}\left(x_{r} \otimes x_{\ell} \otimes\left(\sigma^{-p}\left(x_{\ell}^{\prime}\right)\right)\right), \\
& h\left(h^{\prime}\left(x_{r} \otimes x_{\ell} \otimes\left(\sigma^{-p}\left(x_{\ell}^{\prime}\right)\right)\right)\right)=\sigma^{-s}\left(h^{\prime}\left(x_{r} \otimes x_{\ell} \otimes\left(\sigma^{-p}\left(x_{\ell}^{\prime}\right)\right)\right)\right), \\
& h\left(x_{r} \otimes y_{\ell}\right)=\sigma^{-s}\left(x_{r} \otimes y_{\ell}\right),
\end{aligned}
$$

so in particular $h\left(y_{\ell}\right) \in \mathcal{O}\left(y_{\ell}\right)$, a contradiction.

Corollary 15 (Finitary Ryan's theorem). $k(X)=2$ for every mixing SFT $X$ with a fixed point.

Proof. The fact that $k(X) \geq 2$ follows from the previous theorem and Lemma 7 . To see that $k(X)=2$, assume to the contrary that $k(X)<2$. From $k(X)=0$ it would follow that $\operatorname{Aut}(X)$ contains only powers of the shift, which is evidently false. Assume then that $k(X)=1$ and that $h$ is a single automorphism which commutes with $h^{\prime} \in \operatorname{Aut}(X)$ only if $h^{\prime}$ is a power of the shift. Because $h$ commutes with itself, it follows that $h=\sigma^{i}$ for some $i \in \mathbb{Z}$. But all $h^{\prime} \in \operatorname{Aut}(X)$ commute with $\sigma^{i}$ and so $\operatorname{Aut}(X)$ contains again only powers of the shift, a contradiction.

\section{Conclusions}

We have constructed glider automorphisms $g$ for mixing SFTs $X$ which have a fixed point, and we have applied these glider maps to prove for such $X$ that $k(X)=2$. It seems that our construction of $g$ should generalize to arbitrary mixing SFTs $X$ which do not necessarily have any fixed points. In this case instead of a fixed point $0^{\mathbb{Z}}$ we need to fix some periodic configuration $p \in X$ (i.e. $\sigma^{k}(p)=p$ for some $k \in \mathbb{N}_{+}$) and we consider points $x \in X$ which are finite (in some sense) with respect to $p$ instead of $0^{\mathbb{Z}}$. In light of this it is probable that $k(X)=2$ for all mixing SFTs $X$.

Acknowledgments. The author thanks Ville Salo for helpful discussions concerning these topics. 


\section{References}

1. D. Lind, B. Marcus: An introduction to symbolic dynamics and coding. Cambridge University Press, Cambridge (1995)

2. J. Patrick Ryan: The shift and commutativity. Mathematical systems theory 6 (1-2), 82-85 (1972)

3. V. Salo: Transitive action on finite points of a full shift and a finitary Ryan's theorem. arXiv:1610.05487v2 (2017)

4. V. Salo, I. Törmä: A One-Dimensional Physically Universal Cellular Automaton. In: Kari J., Manea F., Petre I. (eds) Unveiling Dynamics and Complexity. CiE 2017. Lecture Notes in Computer Science, vol 10307. Springer, Cham (2017) 